\title{
Inorganic: Organic Hybrid Nanocomposites for Photorefractivity at Communication Wavelengths
}

\author{
Jeffrey G. Winiarz, Liangmin Zhang, Jooho Park, and Paras N. Prasad* \\ Institute for Lasers, Photonics, and Biophotonics, Departments of Chemistry, Physics, and \\ Electrical Engineering, State University of New York at Buffalo, Buffalo, New York 14260
}

Received: October 15, 2001

\begin{abstract}
This paper reports new photorefractive polymeric nanocomposites photosensitized with $\mathrm{HgS}$ or $\mathrm{PbS}$ nanocrystals and operating at the communication wavelength of $1.3 \mu \mathrm{m}$. To our knowledge, it is the first report of a polymeric photorefractive medium with spectral response at a communication wavelength. The nanocomposites involving $\mathrm{HgS}$ are prepared by an in-situ nanochemistry approach, whereas those involving $\mathrm{PbS}$ were prepared using competitive nanochemistry. Photoconductivity experiments were employed in the characterization of photocharge generation quantum efficiency provided by the semiconductor nanocrystals. The photorefractive nature of the composites is confirmed using electric field dependent two-beam coupling. In the case of nanocomposite containing $\mathrm{PbS}$ nanocrystals, a net gain in excess of the associated absorption loss is observed.
\end{abstract}

\section{Introduction}

Photorefractivity, a multifunctional property derived from the combination of photoconductivity and electrooptic activity, has been the focus of an extensive body of research due to its potential application to real-time optical information processing; beam clean up and amplification, dynamic interferometry, phase conjugation, and pattern recognition. ${ }^{1-3}$ Due to their large optical nonlinearities, low dielectric constants and low cost, polymeric photorefractive (PR) materials in particular have attracted a significant amount of attention during the past 10 years, and tremendous progress has been made in their development. ${ }^{4-19}$ Although many dyes are well suited for visible wavelengths, none have been reported to photosensitize PR polymeric composites at the infrared wavelengths of 1.31 or $1.55 \mu \mathrm{m}$, commonly used in optical communications. Although considerable effort has been made to alter the absorption spectra of recognized organic photosensitizers through functionalization of the parent chromophore, the observed spectral shifts have been meager and unpredictable.

With the advent of nanocrystal (NC) technology, a new means by which to photosensitize polymer composites has been realized, establishing a novel class of inorganic-organic hybrid photoconductive materials. ${ }^{20-22}$ Perhaps the primary advantage gained through this approach concerns the ease with which the spectral properties of NCs are modified, made possible by the quantum size effect where the magnitude of the optical bandgap is inversely proportional to the size of the nanocrystal. ${ }^{23-26}$ This characteristic in conjunction with the wide range of materials available, including narrow band-gap semiconductors, permits this approach to be used for ultra-violet and visible as well as for applications involving infrared wavelengths. In addition, superior photocharge generation efficiency, $\Phi$, associated with inorganic semiconductor materials incorporated into these hybrid composites, may result in a notable PR figureof-merit obtained with a relatively small applied electric field. We recently reported photorefractivity in the visible spectral

* To whom correspondence should be addressed. E-mail: pnprasad@ acsu.buffalo.edu. Tel: (716) 645-6800 ext. 2099. Fax: (716) 645-6945 range using nanocomposites of a polymer, poly $N$-vinylcarbazole (PVK), and CdS nanocrystals. ${ }^{16,17}$

Here, we report on the incorporation of narrow band-gap semiconductor NCs into a PR polymeric matrix to produce, to our knowledge for the first time, photorefractivity at a communication wavelength $(1.3 \mu \mathrm{m}$ in the present case). Two composites were studied; in both cases the photoconductor, PVK, was the primary continuous medium for the transport of the photogenerated charge-carriers (holes). The $T_{\mathrm{g}}$ of the composite was decreased to below ambient temperature through the inclusion of a plasticizer tritolyl phosphate (TCP), allowing for room-temperature electric field alignment of a second-order nonlinear optical (NLO) chromophore, $N$-(4-nitrophenyl)- $(s)$ prolinol (NPP). Although NPP exhibits traditional electrooptic activity (Pockels effect), modulation of the refractive index in the PR operation occurs primarily through birefringence since the chromophore can align with respect to the periodic spacecharge field. ${ }^{13,16}$ The composites were photosensitized at 1.31 $\mu \mathrm{m}$ through the inclusion of nanocrystals composed of lead sulfide $(\mathrm{NCPbS})$ or mercury sulfide $(\mathrm{NCHgS})$. Assuming complete conversion of mercury acetate to $\mathrm{NCHgS}$ and neglecting the weight of the passivating layer associated with the $\mathrm{NCPbS}$, the sample composition was PVK:TCP:NPP:nanocrystal $=50.8: 38.1: 10.2: 0.99$ wt $\%$.

\section{Experimental Section}

For PR samples doped with $\mathrm{NCHgS}$, an in-situ nanochemistry approach was used where fabrication involved solvent casting PVK, TCP, NPP, and mercury acetate on to an indium tin oxide (ITO) coated substrate. Upon complete removal of the solvent, the samples were exposed to $\mathrm{H}_{2} \mathrm{~S}$ converting the mercury acetate to $\mathrm{NCHgS}$ and acetic acid. The byproduct was removed under vacuum, and the sample was subsequently heated above its melting temperature and sandwiched with another ITO coated substrate. By varying the temperature and the time duration of $\mathrm{H}_{2} \mathrm{~S}$ exposure, it is possible to exercise a large degree of control over the eventual size of $\mathrm{NCHgS}$. Transmission electron microscopy as well as X-ray diffraction (XRD) was used to determine particle size; ${ }^{27}$ the average size of $\mathrm{NCHgS}$ in our 


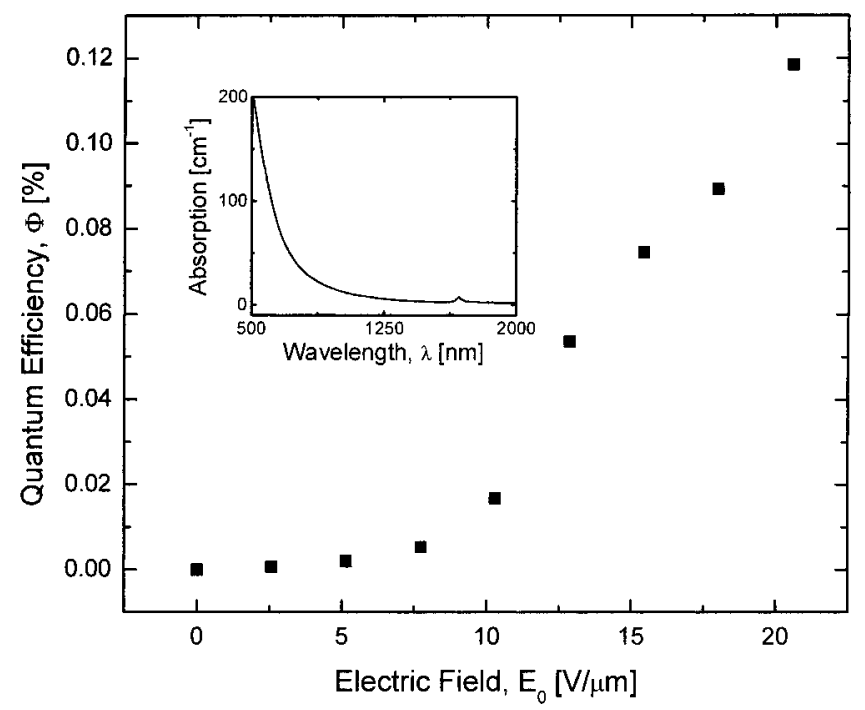

Figure 1. Photocharge generation quantum efficiency, $\Phi$, as a function of applied electric field, $E_{0}$, at $1.31 \mu \mathrm{m}$ for the PVK:TCP:NPP:NCPbS composite. The inset shows the absorption spectrum of the composite.

samples was $\sim 10 \mathrm{~nm}$. In the case of samples containing $\mathrm{NCPbS}$, the nanocrystals were synthesized using wet chemical techniques. Surface passivation of the $\mathrm{NCPbS}$ was accomplished through their encapsulation with a suitable organic moiety, in the present case $p$-thiocresol. Here, the eventual size of the particle was controlled by adjusting the ratio of sulfide $\left(\mathrm{S}^{2-}\right)$ to $p$-thiocresol in solution, thus taking advantage of the competitive reaction rate of these species with solvated lead ions. ${ }^{28}$ Upon purification, the $\mathrm{NCPbS}$ was suspended in an organic solvent, and solvent cast together with PVK, TCP and NPP on to an ITO coated substrate. Upon removal of the solvent, the samples were heated and sandwiched between a second piece of ITO. The mean diameter of $\mathrm{NCPbS}$ was determined to be $\sim 50 \mathrm{~nm}$. The mechanism by which charge transport occurs across the inert passivating layer is the subject of current debate and in recent literature several possibilities have been proposed. ${ }^{13,16,29,30}$ Samples for photoconductive characterizations were similarly fabricated, but here films approximately $5 \mu \mathrm{m}$ in thickness were spin-coated onto an ITO coated substrate and counter electrodes were fabricated via high-vacuum deposition of metallic silver.

\section{Results and Discussion}

Characterization of the photocharge-generating quantum efficiency, $\Phi$, of the nanocrystal:polymer composites at 1.31 $\mu \mathrm{m}$ was accomplished using a simple dc photocurrent technique. The electric field dependencies of $\Phi$ for both composites are depicted in Figures 1 and 2 and were determined according to the equation

$$
\Phi(E)=\frac{N_{\mathrm{cc}}}{N_{\mathrm{ph}}}=J_{\mathrm{ph}}\left(\frac{\mathrm{hc}}{\lambda e \alpha d}\right)
$$

where $N_{\mathrm{cc}}$ is the number of charge carriers generated per unit volume, $N_{\text {ph }}$ is the number of photons absorbed per unit volume in the sample, $J_{\mathrm{ph}}$ is the photocurrent density, $h$ is Planck's constant, $c$ is the speed of light, $\alpha$ is the absorption coefficient of the sample, and $d$ is the sample thickness. An attempt was made to fit the data to the Onsager formalism; ${ }^{17,20,31}$ however, a convergence was not observed indicating the mechanism of charge-generation and subsequent separation to be more complex than that assumed by this model.

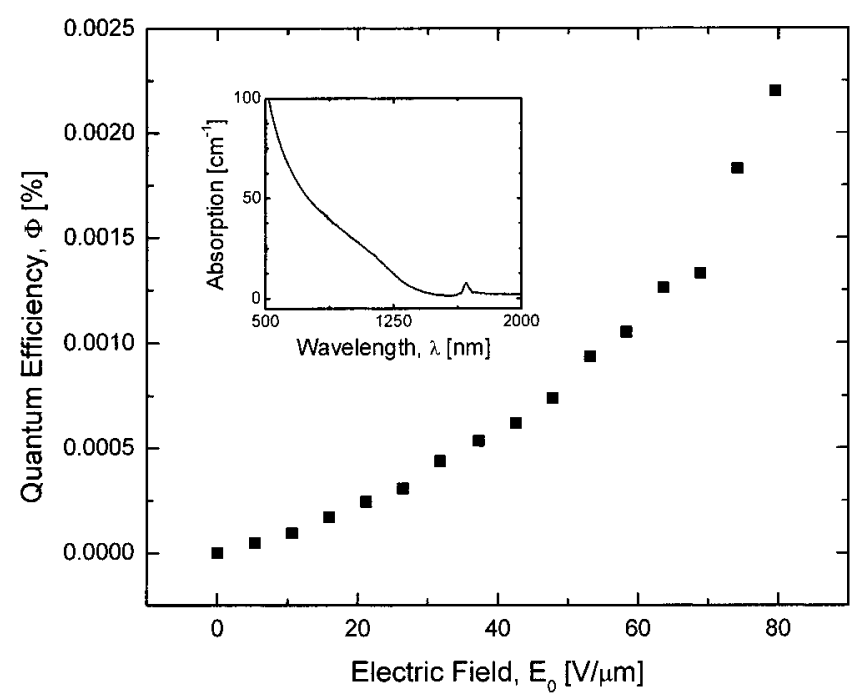

Figure 2. Photocharge generation quantum efficiency, $\Phi$, as a function of applied electric field, $E_{0}$, at $1.31 \mu \mathrm{m}$ for the PVK:TCP:NPP:NCHgS composite. The inset shows the absorption spectrum of the composite.

The PR nature of both composite materials is demonstrated through asymmetric two-beam-coupling (TBC) experiments. Holographic gratings were written by two coherent laser beams from a Lepton IV Series Laser (Micro Laser System, Model \# L41310D-30) operating at $1.31 \mu \mathrm{m}$ with $p$-polarization. The writing beams were of approximately equal intensity and intersected in the samples at incident angles of $60^{\circ}$ and $38^{\circ}$ (in air) respectively, creating an intensity grating with a periodic spacing of $\Lambda=4.75 \mu \mathrm{m}$. The TBC gain coefficient $\Gamma$ is given in terms of the experimentally determined quantities $\gamma_{0}$ and $\beta$, as

$$
\Gamma=\frac{1}{L}\left[\ln \left(\gamma_{0} \beta\right)-\ln \left(\beta+1-\gamma_{0}\right)\right]
$$

where $L$ is the length of the optical path of the beam experiencing gain inside the sample; $\beta$ is the ratio of the writing beam intensities before the sample; and $\gamma_{0}$ is the beam-coupling ratio, defined as $\gamma_{0}=P_{1} / P_{0}$ where $P_{1}$ is the intensity of the signal with the pump, and $P_{0}$ is the intensity of the signal without the pump. ${ }^{3,32,33}$

In Figure 3 the electric field dependence of $\Gamma$ for the $\mathrm{NCPbS}$ sample (thickness $=170 \mu \mathrm{m}$ ) is presented. The solid line is a fit derived from the standard theory of photorefractivity. ${ }^{34-36}$ Here, the magnitude of the space-charge electric field, $\left|E_{\mathrm{sc}}\right|$ takes the form

$$
\left|E_{\mathrm{sc}}\right|=\frac{m E_{\mathrm{q}} \sqrt{E_{0}^{2}+E_{\mathrm{d}}^{2}}}{\sqrt{E_{0}^{2}+\left(E_{\mathrm{d}}+E_{\mathrm{q}}\right)^{2}}} \times \frac{1}{1+\sigma_{\mathrm{d}} / \sigma_{\mathrm{p}}}
$$

where $m=\sqrt{I_{1} I_{2}} /\left(I_{1}+I_{2}\right)$ is the modulation depth; $E_{\mathrm{q}}=e N_{\mathrm{pr}} /$ $\left(\epsilon_{0} \epsilon_{\mathrm{r}} \mathrm{K}_{\mathrm{G}}\right)$ is the trap-density-limited space-charge field, where $N_{\text {pr }}$ is the effective trap density, $\epsilon_{0}$ is the permittivity of free space, $\boldsymbol{K}_{\mathrm{G}}$ is the grating wave vector, and $e$ is the fundamental unit charge; $E_{\mathrm{d}}=k_{\mathrm{B}} T \mathrm{~K}_{\mathrm{G}} / e$ is the diffusion field, where $k_{\mathrm{B}}$ is Boltzmann's constant and $T$ is the absolute temperature; and $\sigma_{\mathrm{p}}$ and $\sigma_{\mathrm{d}}$ are photoconductivity and dark conductivity, respectively. Assuming the general condition $E_{\mathrm{d}} \ll E_{0}, E_{\mathrm{q}}$ is met, $\Gamma$ can be expressed as

$$
\Gamma=\Gamma_{0} \sin \phi_{\mathrm{p}}, \Gamma_{0}=\frac{2 \pi}{\lambda} n^{3} R E_{0}\left|\frac{E_{\mathrm{sc}}}{m}\right|,
$$




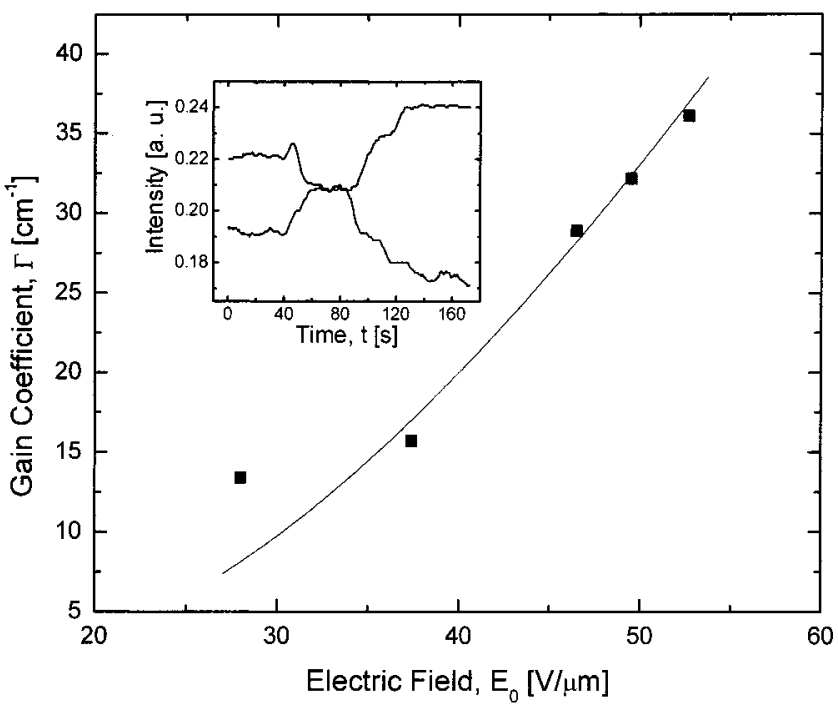

Figure 3. Electric field dependence of the TBC gain coefficient $\Gamma$ in the PVK:TCP:NPP:NCPbS composite at $1.31 \mu \mathrm{m}$. The line corresponds to a theoretical fit (see text). The inset shows asymmetric energy exchange, here, the external electric field $\left(E_{0}=52.7 \mathrm{~V} / \mu \mathrm{m}\right)$ was applied at $t=45 \mathrm{~s}$ and turned off at $t=90 \mathrm{~s}$.

where $\phi_{\mathrm{p}}=\arctan \left(\left(E_{0}^{2}+E_{\mathrm{d}} E_{\mathrm{q}}\right) / E_{0} E_{\mathrm{q}}\right)$ is the phase of the grating vector, $\lambda$ is the wavelength of the incident radiation, $n$ is the refractive index, and $R$ is a coefficient describing the effective NLO activity including the birefringence as well as the traditional electrooptic contributions. As is evident in the figure, a reasonably good theoretical fit is realized for the data and a value of $N_{\mathrm{pr}}=1.06 \times 10^{17} \mathrm{~cm}^{-3}$ is obtained as the effective trap density. The maximum TBC gain coefficient, measured just prior to the dielectric breakdown of the sample, was $\Gamma=36.1$ $\mathrm{cm}^{-1}$ and the magnitude of the space charge field, $E_{\mathrm{sc}}$, was 18.8 $\mathrm{V} / \mu \mathrm{m}$ where $E_{0}=52.7 \mathrm{~V} / \mu \mathrm{m}$ (corrections were made for reflection at the glass/air interface and for the absorption attributed to ITO). From a practical point of view, the optical amplification, $\Gamma$, must exceed the absorption loss, $\alpha$, of the PR sample in question. ${ }^{37-39}$ In this case, the optical loss of the sandwiched sample (glass/ITO/polymer composite/ITO/glass) at $1.31 \mu \mathrm{m}$ was measured to be $\alpha=4.2 \mathrm{~cm}^{-1}$ yielding a maximum net gain coefficient of $\Gamma-\alpha=30.9 \mathrm{~cm}^{-1}$. An example of asymmetric exchange of energy is depicted in the inset in Figure 3, which confirms the PR nature of the NCPbS composite. Also evident in the figure is that when the external electric field is removed from the sample, the intensities of the writing beams after the sample do not return to exactly their original values, indicating the presence of a secondary grating. While uniform illumination of the sample does not affect the secondary grating, it is reversible in that it is observed to decay over a time span of $\sim 30 \mathrm{~min}$. The mechanism responsible for this secondary grating is the subject of current studies.

The electric field dependence of TBC gain coefficient, $\Gamma$, for the NCHgS sample (thickness $=155 \mu \mathrm{m}$ ) is illustrated in Figure 4. The data presented here was as well fit to eqs 3 and 4 yielding an effective trap density of $N_{\mathrm{p} r}=9.25 \times 10^{16} \mathrm{~cm}^{-3}$, again producing a good fit of the data. Here it was calculated that an electric field of $E_{0}=90 \mathrm{~V} / \mu \mathrm{m}$ produced a space charge field, $E_{\mathrm{sc}}$, of $21.0 \mathrm{~V} / \mu \mathrm{m}$ and yielded a TBC gain coefficient of $\Gamma=4.35 \mathrm{~cm}^{-1}$. Unlike the $\mathrm{NCPbS}$ composite, the optical amplification associated with the composite doped with $\mathrm{NCHgS}$ did not exceed that of the absorption losses where $\alpha=9.48$ $\mathrm{cm}^{-1}$. Graphical illustration of the asymmetric exchange of

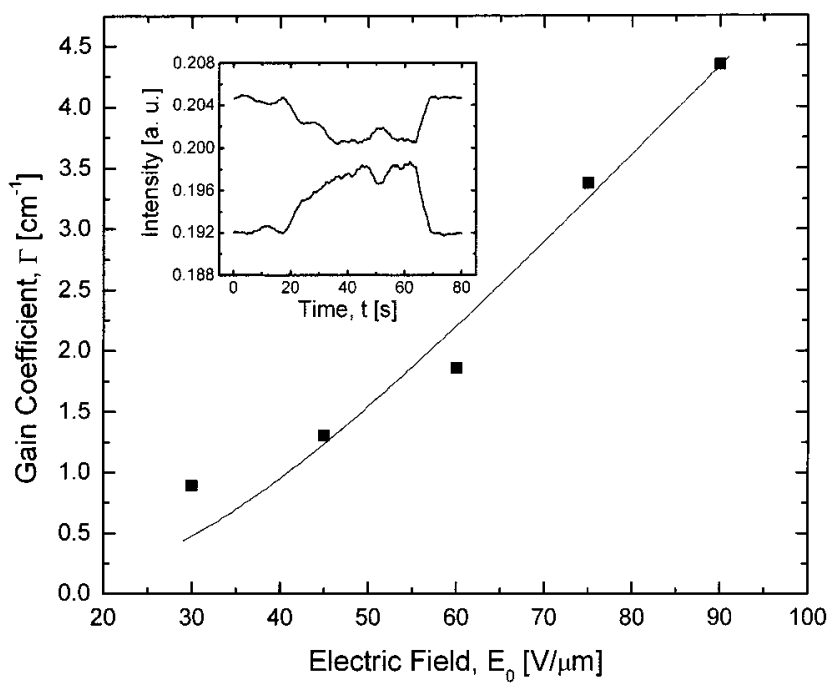

Figure 4. Electric field dependence of the TBC gain coefficient $\Gamma$ in the PVK:TCP:NPP:NCHgS composite at $1.31 \mu \mathrm{m}$. The line corresponds to a theoretical fit (see text). The inset shows asymmetric energy exchange, here, the external electric field $\left(E_{0}=90 \mathrm{~V} / \mu \mathrm{m}\right)$ was applied at $t=17 \mathrm{~s}$ and turned off at $t=63 \mathrm{~s}$.

energy in the case of the $\mathrm{NCHgS}$ sample is presented in the inset of Figure 4, thereby confirming the PR nature of this composite.

\section{Conclusion}

In summary, it has been demonstrated that inorganic nanocrystals can be effectively used to photosensitize PR polymeric composites for operation at the infrared wavelength of $1.31 \mu \mathrm{m}$ commonly used in telecommunications. Moreover, the quantum efficiency of the photocharge generation process associated with $\mathrm{NCPbS}$ or $\mathrm{NCHgS}$ doped composites has been characterized at this wavelength.

Acknowledgment. We acknowledge Dr. R Burzynski of Laser Photonics Technology, Inc. and Dr. Marek Samoc for their help. This work was supported in part by the Directorate of Chemistry and Life Sciences of the Air Force Office of Scientific Research Grant No. F496209610124, a DOD nanotechnology initiative DURINT Grant No. (F496200110358) and in part by a NSF, DMR Solid state and polymer chemistry Grant No. (DMR0075867).

\section{References and Notes}

(1) Photorefractive Materials and Their Applications, I \& II, Topics in Applied Physics, Vols. 61 and 62; Gunter, P., Huignard, J.-P., Eds.; Springer-Verlag: Berlin, 1988

(2) Yeh, P. Introduction to Photorefractive Nonlinear Optics; Wiley: New York, 1993.

(3) Morner W. E.; Silence, S. M. Chem. Rev. 1994, 94, 127-55.

(4) Ducharme, S.; Scott, J. C.; Twieg, R. J.; Moerner, W. E. Phys. Rev. Lett. 1991, 66, 1846-9.

(5) Cui, Y.; Zhang, Y.; Prasad, P. N.; Schildkraut, J. S.; Williams, D. J. Appl. Phys. Lett. 1992, 61, 2132-4.

(6) Zhang, Y.; Cui, Y.; Prasad, P. N. Phys. Rev. B: Condens. Matter 1992, 46, 9900-2.

(7) Volodin, B. L.; Kippelen, B.; Meerholz, K.; Javidi, B.; Peyghambarian, N. Nature (London) 1996, 383, 58-60.

(8) Orczyk, M. E.; Zieba, J.; Prasad, P. N. J. Phys. Chem. 1994, 98 , 8699-704.

(9) Orczyk, M. E.; Swedek, B.; Zieba, J.; Prasad, P. N. J. Appl. Phys. 1994, 76, 4995-8.

(10) Orczyk, M. E.; Zieba, J.; Prasad, P. N. Appl. Phys. Lett. 1995, 67, $311-3$.

(11) Peng, Z.; Gharavi, A.; Yu, L. Polym. Prepr. 1995, 36, 41-2. 
(12) Cheng, N.; Swedek, B.; Prasad, P. N. Appl. Phys. Lett. 1997, 71, $1828-30$.

(13) Swedek, B.; Cheng, N.; Cui, Y.; Zieba, J.; Winiarz, J.; Prasad, P. N. J. Appl. Phys. 1997, 82, 5923-31.

(14) Cui, Y.; Swedek, B.; Cheng, N.; Kim, K.-S.; Prasad, P. N. J. Phys Chem. B 1997, 101, 3530-4.

(15) Cui, Y.; Swedek, B.; Cheng, N.; Zieba, J.; Prasad, P. N. J. Appl. Phys. 1999, 85, 38-43.

(16) Winiarz, J. G.; Zhang, L.; Lal, M.; Friend, C. S.; Prasad, P. N. J. Am. Chem. Soc. 1999, 121, 5287-95.

(17) Winiarz, J. G.; Zhang, L.; Lal, M.; Friend, C. S.; Prasad, P. N. Chem. Phys. 1999, 245, 417-28.

(18) Burzynski, R.; Kumar, D. N.; Casstevens, M. K.; Tyczka, D. Ghosal, S.; Kurtz, P. M.; Weibel, J. F. Proc. SPIE-Int. Soc. Opt. Eng. 2000 4087, 741-53.

(19) Wang, L.; Ng, M.-K.; Yu, L. Appl. Phys. Lett. 2001, 78, 700-2.

(20) Herron, N.; Wang, Y. US Patent 5,238,607, 1993.

(21) Wang, Y.; Herron, N. Chem. Phys. Lett. 1992, 200, 71.

(22) Wang, Y. Kirk-Othmer Encyclopedia of Chemical Technology, Fourth Edition; Kroschwitz, J., Ed.; Wiley: New York, 1996.

(23) Steigerwald, M. L.; Brus, L. E. Acc. Chem. Res. 1990, 23, 183.

(24) Nosaka, Y.; Ohta, N.; Miyama, H. J. Phys. Chem. 1990, 94, $3752-$ 5 .

(25) Wang, Y.; Herron, N. J. Phys. Chem. 1991, 95, 525.

(26) Wang, Y. Advances in Photochemistry, Vol. 19; Neckers, D. C.,
Volman, D. H., Von Bünau, G., Eds.; John Wiley and Sons: New York, 1995.

(27) Klug, H. P.; Alexander, L. E. X-ray Diffraction Procedure for Polycrystalline and Amorphous Materials, 2nd ed.; Wiley: New York, 1974.

(28) Herron, N.; Wang, Y.; Eckert, H. J. Am. Chem. Soc. 1990, 112, $1322-6$

(29) Greenham, N. C.; Xiaogang, P.; Alivisatos, A. P. Physical Review 1996, 54, 17 628-37.

(30) Nanda, J.; Narayan, K. S.; Kuruvilla, B. A.; Murthy, G. L.; Sarma, D. D. Appl. Phys. Lett. 1998, 72, 1335-7.

(31) Onsager, L. Phys. Rev. 1938, 54, 554.

(32) Yeh, P. IEEE. J. Quan. Electronic 1989, 25, 484-519.

(33) Donckers, M. C. J. M.; Silence, S. M.; Walsh, C. A.; Hache, F.; Burland, D. M.; Morner, W. E.; Twieg, R. J. Opt. Lett. 1993, 18, 1044-6. (34) Kukhtarev, N. V.; Markov, V. B.; Odulov, S. G.; Soskin, M. S.; Vinetskii, V. L. Ferroelectrics 1979, 22, 949.

(35) Ducharme, S. Proc. SPIE 1995, 2526, 144-54.

(36) Grunnet-Jepsen, A.; Thompson, C. L.; Twieg, R. J.; Moerner, W. E. Appl. Phys. Lett. 1997, 70, 1515-7.

(37) Donckers, M. C. J. M.; Silence, S. M.; Walsh, C. A.; Hache, F.; Burland, D. M.; Morner, W. E.; Twieg, R. J. Opt. Lett. 1993, 18, 1044-6. (38) Liphardt, M.; Goonesekera, A.; Jones, B. E.; Ducharme, S.; Takacs, J. M.; Zhang, L. Science 1994, 263, 367-9.

(39) Zhao, C.; Park, C.; Prasad, P. N. Chem. Mater. 1995, 7, 1237-42. 